\title{
Magnetoresistance of Ferromagnetic Point Junctions From Tunneling to Direct Contact Regimes
}

\author{
H. Pandana, L. Gan, M. Dreyer, C. Krafft, and R. D. Gomez
}

\begin{abstract}
The behavior of magnetoresistance in $\mathrm{NiFe}$ (permalloy) point junctions was investigated using an STM set-up, where the junction resistance was continuously varied from $10^{8} \Omega-10^{3} \Omega$. Our results reveal an enhancement of magnetoresistance of more than $80 \%$ at one conductance quantum, and the decaying magnetoresistance as one moves away from $12.9 \mathrm{k} \Omega$ to either the diffusive regime or the tunneling regime, as supported by independent theories on spin-dependent transport. The suppression of magnetoresistance with incorporation of a 35-nm-thick Au barrier was observed, as well as the dependence of magnetoresistance on the relative orientations of the electrodes.
\end{abstract}

Index Terms-Ballistic magnetoresistance, magnetoresistance, scanning tunneling microscope (STM).

\section{INTRODUCTION}

$\mathbf{M}$ AGNETORESISTIVE (MR) effects are the backbone of data storage systems, memory devices, and magnetic field sensors. Tunneling magnetoresistance (TMR) appears in ferromagnet-insulator-ferromagnet multilayers in the resistance range of several hundred kilo-ohms to megaohms depending on the junction area. Giant magnetoresistance is exhibited in ferromagnetic films sandwiching a normal metal, and anisotropic magnetoresistance (AMR) exists in single ferromagnetic layers with resistances as small as a few ohms. Mathon [1] developed a unified theory of the TMR and the ballistic-current perpendicular-to-plane giant magnetoresistance to predict the behavior of cobalt junctions in the tunneling and metallic regimes. Similarly, Tatara et al. [2] explained the behavior of magnetoresistance of nanoconstricted wires and the enhancement of MR at one resistance quantum $\left(R_{0}=\left(2 e^{2} / h\right)^{-1}=12.9 \mathrm{k} \Omega\right)$ commonly known as ballistic MR (BMR). This enhancement is attributed to spin scattering in the narrow domain wall (DW) formed at the constricted region. BMR has been observed by several groups [3]-[5] and Chung et al. [6] further provided a universal scaling to all MR versus resistance data and found that they fall within a narrow region predicted by theory.

In this work, our interest is in understanding the evolution of MR from a tunneling to a direct contact in a single experimental setup. Along with the behavior at both the low and high resistance limits, we observed the enhancement of MR

Manuscript received October 16, 2003. This work was supported in part by NSF ECS 0115327 (HP, RDG).

H. Pandana, L. Gan, M. Dreyer, and R. D. Gomez are with the Departmet of Electrical and Computer Engineering, University of Maryland, College Park, MD 20742 USA (e-mail: hpandana@glue.umd.edu; gan@glue.umd.edu; dreyer@1ps.umd.edu; rdgomez@ece.umd.edu).

C. Krafft is with the Laboratory for Physical Sciences, College Park, MD 20742 USA (e-mail: krafft@1ps.umd.edu).

Digital Object Identifier 10.1109/TMAG.2004.829833 near $12.9 \mathrm{k} \Omega$, as predicted by the theories [1], [2] using completely different approaches. We further established the veracity of spin-polarized transport as the mechanism for MR enhancement by studying the suppression of the effect as a function of gold overlayer thickness and magnetization orientation.

\section{EXPERIMENT}

A scanning tunneling microscope (STM) was used for this study. An electrochemically etched $\mathrm{Ni}_{80} \mathrm{Fe}_{20}$ (NiFe) wire mounted inside an electromagnet was used as the tip. $\mathrm{NiFe}$ was chosen because of its low coercivity and very low magnetostriction. Prior to use, the tip was ion-sputtered to remove the oxide deposit and capped with $10 \mathrm{~nm}$ of $\mathrm{Au}$ in UHV. The tip radius after processing was about $0.5 \mu \mathrm{m}$. The electromagnet was driven by an ac current and modulated the tip magnetization between positive and negative saturation. The experiment was initiated as a regular STM, where the tip slowly approached the surface until a tunneling current was detected. A $z$-axis piezo, connected to the output of an electronic feedback circuit, ensured that the tip-sample distance maintained a predetermined setpoint tunneling current. To perform the MR measurements, we used a slow feedback response to eliminate the system's ability to track rapid variations in the tunneling current. We then measured the change in current at a fixed voltage of $200 \mathrm{mV}$ as the tip magnetization was modulated between positive and negative saturation at $f \sim 300 \mathrm{~Hz}$. The magnetoresistance ratio is defined to be the absolute value of the difference in the current when the tip is fully magnetized one way and the opposite, divided by the smaller current, and can exceed $100 \%$. The lateral scan was disabled during the run so that a specific local area of the surface (within drift tolerance) was studied. The tip-sample resistance was set by selecting a current setpoint, which is tantamount to the adjustment of the tip-sample distance. As the tip is often damaged after closest approach, we always used new tips and varied the resistance from high to low values. All measurements were done at room temperature and ambient pressure.

Several samples were studied: a polished face of 1-in NiFe rod, which was ion-sputtered to remove the native oxide layer and capped with gold $(10 \mathrm{~nm})$; a polished surface of NiFe rod without ion-sputtering; and a $30-\mathrm{nm} \mathrm{NiFe}$ thin film on $\mathrm{SiO}_{2}$. The thin film sample was not mounted on the electromagnet.

\section{RESULTS AND DISCUSSIONS}

The behavior of magnetoresistance as a function of junction resistance for a saturated $\mathrm{NiFe}$ rod is shown in Fig. 1. Each data point represents the average of $\sim 1000$ cycles while the 


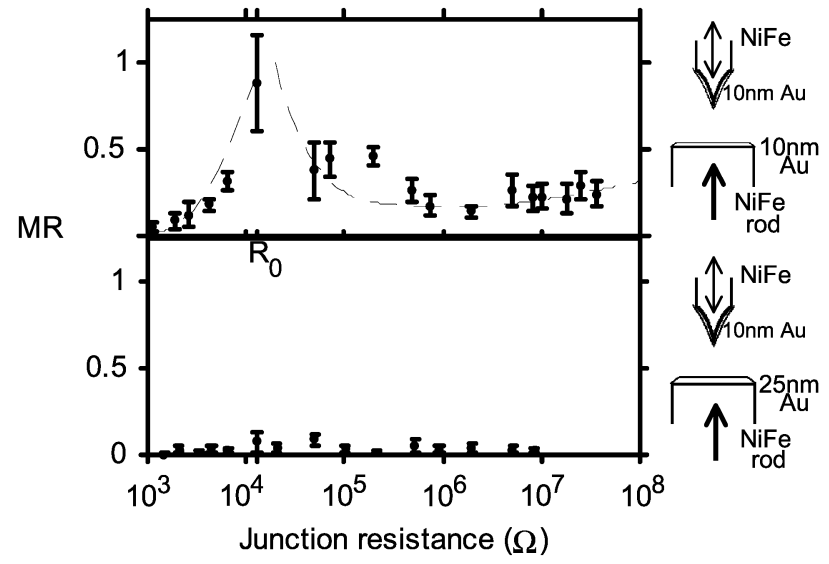

Fig. 1. MR versus $\mathrm{R}$ curves for $\mathrm{NiFe}$ wire and $\mathrm{NiFe}$ rod. (Top) Both $\mathrm{NiFe}$ wire and $\mathrm{NiFe}$ rod were ion-sputter cleaned and coated with 10-nm gold. The dashed lines are qualitative trends predicted by the theories. (Bottom) Massive suppression of MR as gold coating on $\mathrm{NiFe}$ rod was increased to $25 \mathrm{~nm}$. These gold thicknesses are nominal thicknesses.

tip is held in place above the sample, and the error bars provide a measure of the data spread. The top plot is data from the 10-nm gold-coated $\mathrm{NiFe}$ rod. At the highest junction resistance $\left(R>10^{7} \Omega\right)$, the curve approaches a value of $25 \% \mathrm{MR}$, which is comparable to the MR value of planar magnetic tunnel junctions. At the other extreme $\left(R<10^{3} \Omega\right)$, the MR is to less than $1 \%$, which indicates the onset of AMR when the tip has made direct contact with the sample. If the magnetization switched exclusively from $0^{\circ}$ to $180^{\circ}$ with respect to the current direction, then we would expect no AMR response to arise. Hence the residual AMR observed at the lowest resistance suggests a contribution from an in-plane magnetization component of the sample.

The more exciting result is the enhancement of MR at one conductance quantum, $R_{0}=12.9 \mathrm{k} \Omega$, which reached up to $80 \%$, and the behavior on either side of this maximum. Mathon explained the MR enhancement in the case of $f c c$ Co (001) in the limit of the metallic regime and an abrupt DW by calculating the transmission coefficient through the junction of each spin channel using a realistic tight binding band structure. According to the model, there is an evolution in the proportion of the current from being mostly carried by weakly spin polarized $s-p$ electrons in the tunneling regime, to being mostly carried by the highly spin polarized $d$ electrons in metallic regime. One, therefore, expects the MR enhancement in the metallic regime limit. Similarly, the behavior of magnetoresistance in the ballistic regime $\left(R \leq R_{0}\right)$ has been explained by Tatara et al. [2] using the Cabrera-Falicov theory [7] of scattering of spin polarized electrons by a domain wall. According to the theory, in thick DWs the electron spin can align with the local magnetization as it traverses the wall. For very thin DWs, however, the spin is almost completely conserved as the electron traverses the wall, so that the MR reduces to the Julliere formula for magnetoresistance. Bruno et al. [8] showed that the DW widths are determined by the size of the constriction which becomes a minimum in case of a single channel ballistic transport. The result is also consistent with the theory of Nakanishi and Nakamura [9] who pointed out that the conductance quantization unit in
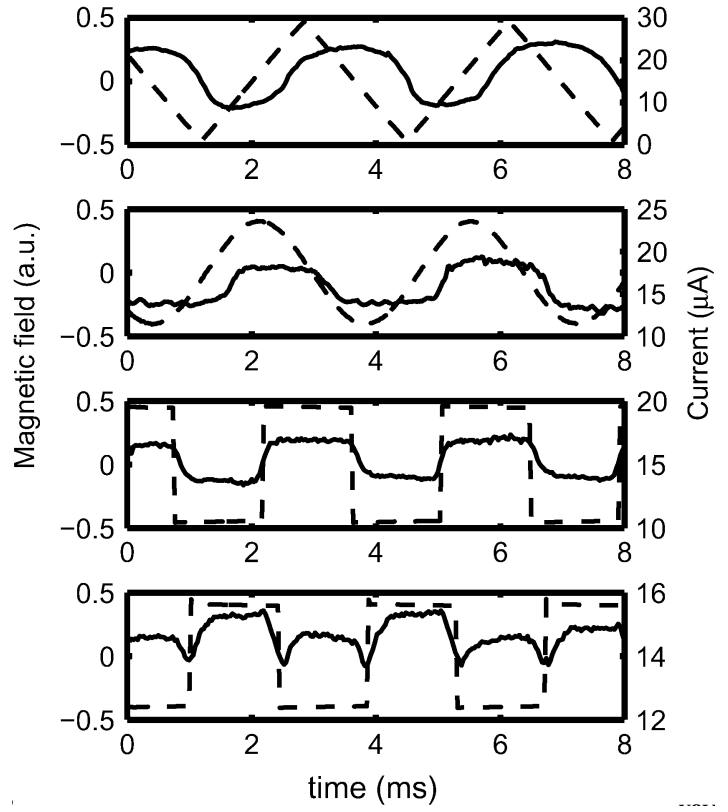

Fig. 2. Typical oscilloscope traces from different applied wave forms. The dashed curves, corresponding to the left axes are the wave forms. The solid curves, corresponding to the right axes, are the current responses $\left(\mathrm{U}_{\text {bias }}=\right.$ $200 \mathrm{mV}$ ). The bottom plot is the response where magnetostriction effect was not removed.

the presence of a domain wall is $2 e^{2} / h$, instead of $e^{2} / h$ as one might expect when the spin degeneracy is lifted [10].

The $R<R_{0}$ portion of the dashed curve in Fig. 1 represents a fit to the theory of Tatara; while the $R>R_{0}$ portion of it was qualitatively fitted to the numerical curve of Mathon. The relationship between our data and the plot generated using the latter model is rather tenuous, but it is nevertheless compelling that the data appear to follow the predictions of the theory obtained using completely different models. The suppression of magnetoresistance by adding $15 \mathrm{~nm}$ of $\mathrm{Au}$ on the sample surface, shown in bottom half of Fig. 1, is also noteworthy. The fact that the 35-nm Au barrier eliminates the effect strongly supports our assertion that the observed resistance changes are mainly due to the coherent transport of spin polarized electrons. Zhang and Levy [11] predicted a long decay length $(\sim 10 \mathrm{~nm})$ of the magnetoresistance when a perfectly flat nonmagnetic layer is inserted between the magnetic electrode and the barrier. The discrepancy between their prediction and our result could perhaps be attributed to deposition conditions. Since no direct film thickness measurement was made, the actual gold thickness may deviate from the $35-\mathrm{nm}$ nominal value and could be close to $10 \mathrm{~nm}$, given the sharp geometry of the tip. The flatness requirement was achieved by polishing and by the smallness of the junction area.

There are legitimate concerns particularly on claims of fantastically high magnetoresistance ratios in the ballistic regime. For example, the simple electrical shorting of the electrodes due to elongation by magnetostriction or magnetostatic force, can be mistaken for spin dependent effects. In those cases, the resistance ratio can approach infinity. In our experiments, we argue against these possibilities with the following observations. By examining the oscilloscope traces in Fig. 2, one can conclude 

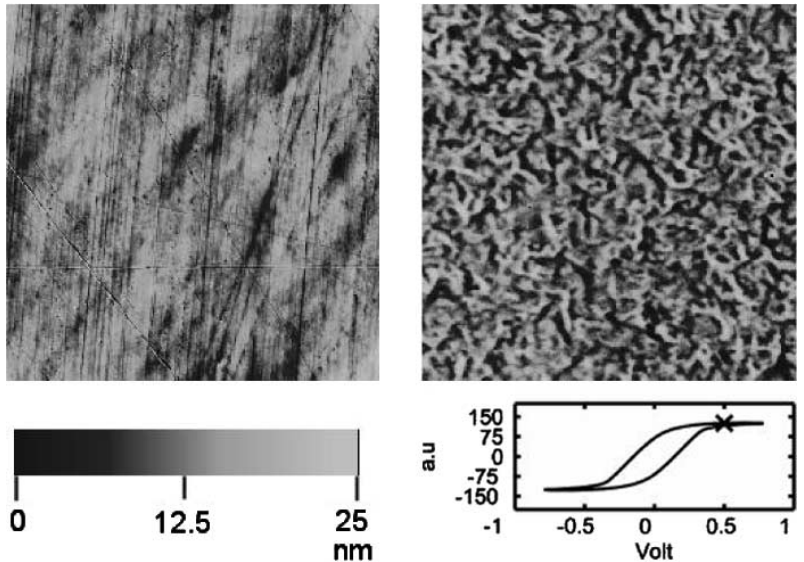

Fig. 3. (Left) AFM image of the polished surface of NiFe rod. (Scan size: $50 \mu \mathrm{m}$.) (Right) The corresponding MFM image with an applied field of magnitude marked by an $\mathrm{X}$ in the hysteresis loop. (Bottom Right) The hysteresis loop measured by a hall probe positioned at the front end of the NiFe rod as a function of voltage applied across the coil.

that a $2 f$ response, which is the signature of magnetostriction, is not observed in the first three traces. The magnetostriction was avoided by firmly attaching the tip to the holder with crystalbond ${ }^{\mathrm{TM}}, 1$ and keeping the protuding part of the tip as short as $3 \mathrm{~mm}$. In contrast, the bottom trace was obtained using a tip without solid mounting and the result shows the characteristic $2 f$ response. One can also easily disregard Faraday induction, since the driving frequency was kept below $300 \mathrm{~Hz}$. It is, however, prudent to point out that STM assembly contains some magnetic materials which may be affected by the coil and cause mechanical motion. Nevertheless, the fact that we found a suppression of magnetoresistance with 35-nm gold interlayer (Fig. 1) suggests that the observed effect in the top plot could not be due to the magnetostatic force between the tip and the rod or other mechanical perturbations. The additional 15-nm spacing cannot diminish the magnetostatic force enough to suppress the resistance change.

Despite careful sample preparation and experimentation, we found a significant spread in the data points. This may be attributable to the intrinsic lack of precise control over the morphological and magnetic configurations. In Fig. 3 we show the topography and magnetic structure of the polished NiFe rod. We observe that even with an applied field close to the saturation field, the MFM image shows a complex multidomain structure at the end of the rod. Hence, it is possible that the tip could have drifted during a measurement and inadvertently was on a region giving little or no MR response such as domain walls. In addition, since the end domains could contain a significant transverse magnetization component, the measured MR response may represent a lower bound.

Finally, we address the angle dependence of MR. The top plot of Fig. 4 is essentially the same as shown in Fig. 1, but measured without the Au film. It corresponds to a polar angle between the tip and sample magnetization of $\theta=0^{\circ}$ and it mimics the earlier plot, except that MR at $R_{0}$ is reduced due to degradation of spin

\footnotetext{
${ }^{1}$ A temporary adhesive that exhibits high bond strength and can be dissolved
} by applying heat (Electron Microscopy Sciences).

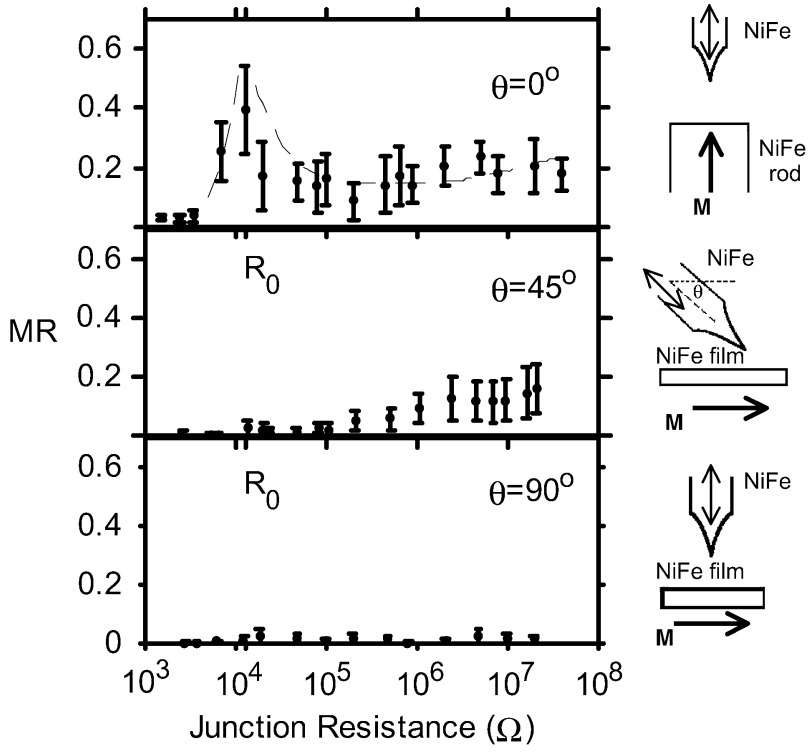

Fig. 4. Polar angle dependence of the MR. The angle $\theta$ denotes the angle between the magnetizations of the two electrodes.

ballisticity inside the junction due to the oxide barrier. In contrast, the middle plot corresponds to $\theta=45^{\circ}$ inclination of the tip over a $30-\mathrm{nm} \mathrm{NiFe}$ film on $\mathrm{SiO}$ substrate. The sample in this case has in-plane magnetization. The TMR is somewhat lower than that of $\theta=0^{\circ}$. This is not surprising, as the expected resistance is $R=R_{0}(1+\epsilon \cos (\theta))$ [12]. But more significantly, the BMR peak is clearly suppressed. The reason is that the electron wave vector is no longer conserved as it traverses the junction, which implies that the transport is no longer ballistic. In the case of a metallic junction, the requirement for spin-ballisticity is as strict as that for transport ballisticity [6], so that BMR is consequently lost. The bottom plot is the MR measurement of the untilted NiFe wire and the in-plane 30-nm NiFe film on $\mathrm{SiO}$ substrate, thereby making a $90^{\circ}$ angle with the sample plane. The suppression of MR for all regimes is evident. If we assume that the tip magnetization at the last domain is exclusively along the axial direction, then the suppression is understandable since the projection of the magnetization from one electrode onto the other is zero.

\section{REFERENCES}

[1] J. Mathon, Phys. Rev. B, vol. 56, p. 11810, 1997

[2] G. Tatara, Y. W. Zhao, M. Muñoz, and N. García, Phys. Rev. Lett., vol. 83, p. 2030, 1999.

[3] N. García, M. Muñoz, and Y. W. Zhao, Phys. Rev. Lett., vol. 8, p. 2923, 1999.

[4] S. Z. Hua and H. D. Chopra, Phys. Rev. B, vol. 67, p. 060401, 2003.

[5] J. J. Verluijs, M. A. Bari, and J. M. D. Coey, Phys. Rev. Lett., vol. 87, p. 026601, 2001.

[6] S. H. Chung, M. Muñoz, N. García, W. F. Egelhoff, and R. D. Gomez, Phys. Rev. Lett., vol. 89, p. 287203, 2002.

[7] G. G. Cabrera and L. M. Falicov, Phys. Status Solidi B, vol. 61, p. 539, 1974.

[8] P. Bruno, Phys. Rev. Lett., vol. 83, p. 2425, 1999.

[9] K. Nakanishi and Y. O. Nakamura, Phys. Rev. B, vol. 61, p. 11278, 2000.

[10] F. Komori and K. Nakatsuji, Mater. Sci. Eng. B, vol. 84, no. 1-2, p. 102, 2001.

[11] S. Zhang and P. M. Levy, Phys. Rev. Lett., vol. 81, p. 5660, 1998.

[12] J. C. Slonczewski, Phys. Rev. B, vol. 39, p. 6995, 1989. 